\title{
Upregulation of miR-137 Expression Suppresses Tumor Growth and Progression via Interacting with DNMT3a Through Inhibiting the PTEN/Akt Signaling in HCC
}

This article was published in the following Dove Press journal: OncoTargets and Therapy

Jiachen Wang

Zhao Wang

Jiaxiang Yuan

Qun Wang

Xinsheng Shen

Department of Minimally Invasive Surgery, The First Affiliated Hospital of

Zhengzhou University, Zhengzhou 450052, Henan, People's Republic of China
Correspondence: Jiachen Wang Department Of Minimally Invasive Surgery, The First Affiliated Hospital of Zhengzhou University, No. I Jianshe East Road, Zhengzhou 450052, Henan,

People's Republic of China

Tel +86037I67967387

Email wangjiachen82@126.com
Background: Downregulation of miR-137 regulates tumor growth in hepatocellular carcinoma (HCC). Yet, the underlying molecular mechanisms stay unclear.

Materials and Methods: miR-137 and DNA methyltransferase 3a (DNMT3a) expression levels were detected by Western blot, immunohistochemistry and qRT-PCR assays. Luciferase reporter and Western blot assays were also carried out to explore the correlation of miR-137 and DNMT3a. Flow cytometry assay, MTT analysis, transwell and wound healing assay were used to evaluate cell apoptosis, proliferation, as well as invasive and migratory abilities. Western blot was used to examine the caspase-3, cleaved caspase-3, PCNA, MMP-2, and MMP-7 protein levels, as well as PTEN/Akt signaling alternations. Methylation-specific PCR was applied to detect the PTEN promoter methylation status. Xenograft tumor assay, Western blot and immunohistochemistry analyses were taken to confirm the miR-137 regulation in vivo.

Results: Downregulation of miR-137, upregulation of DNMT3a, as well as an inverse correlation between them were observed in HCC clinical samples and cells. Moreover, miR-137 targeted directly and inhibited DNMT3a in HCC cells, which further retarded cell proliferative, migratory and invasive capabilities, while promoted apoptotic ones. Additionally, miR-137 overexpression inactivated the PTEN/Akt pathway in HCC cell by decreasing DNMT3a expression. Furthermore, miR-137 overexpression inhibited tumor growth in vivo in HCC via interacting with DNMT3a through inhibiting the PTEN/Akt cascades.

Conclusion: Our findings suggested that miR-137 inhibited HCC tumor growth and progression via interacting with DNMT3a and suppressing the PTEN/Akt signaling in vitro and in vivo. Keywords: miR-137, DNMT3a, DNA methylation, hepatocellular carcinoma, the PTEN/ Akt pathway

\section{Introduction}

Hepatocellular carcinoma (HCC), belonging to the commonest diagnosed carcinomas, makes up the second highest carcinoma-associated deaths with rapidlyincreasing incidence and postoperative recurrence worldwide. ${ }^{1}$ According to cancer statistical data in 2017, there were approximately 40,710 cases and 28,920 deaths due to HCC in the USA. ${ }^{1}$ To date, hepatic transplantation, resection, and chemotherapy are common traditional methods for the treatment of early-stage HCC. ${ }^{2}$ However, the advanced stage cases were counted most among the diagnosed ones due to the deficiency of distinct HCC biomarkers. ${ }^{3}$ In spite of the recent advances in 
fighting against $\mathrm{HCC}$, the clinical outcomes remain unfavorable with only $15 \% 5$-year overall survival rate. $^{4}$ Hence, crystalizing the regulation and exploring novel effective treatments for HCC are in great need. microRNAs (miRNAs), small single-stranded non-coding RNAs, post-transcriptionally regulate the gene expression. ${ }^{5}$ Increasing experimental data have reported the association between miRNAs and pathological cellular processes (ie, proliferation, growth, cell cycle, apoptosis, and metastasis). ${ }^{6}$ It has been implicated that dysregulation of miRNAs is frequent in diverse carcinomas including HCC. $^{7}$ Moreover, miRNAs are well documented to be closely implicated in either promoting or suppressing HCC carcinogenesis. ${ }^{8}$ Among these miRNAs, miR-137 has been confirmed to act as a tumor suppressor and be underexpressed in various carcinomas. ${ }^{9-11}$ Furthermore, studies also have implicated that the downregulation of miR-137 has a regulatory effect on HCC tumorigenesis. ${ }^{12}$ However, the detailed mechanisms are not well elucidated.

Herein, our results confirmed the downregulation of miR-137 and observed upregulation of DNA methyltransferase 3a (DNMT3a), a miR-137 target, in HCC patients and cells. Mechanistically, overexpressing miR-137 inhibited HCC carcinogenesis via interacting with DNMT3a and suppressing the PTEN/Akt signaling. Our reports would provide great worth for understanding hepatocarcinogenesis mechanisms better.

\section{Materials and Methods Clinical Samples}

HCC and paired peritumoral healthy tissues were obtained from twenty-nine HCC patients undergoing hepatectomy at the First Affiliated Hospital of Zhengzhou University from July 2017 to June 2018. After surgical resection, all tissues were immediately snap-frozen for RNA extraction. None of the involved patients accepted chemotherapy and/ or radiotherapy prior to the surgery. The study protocols were approved by the Ethics Committee of First Affiliated Hospital of Zhengzhou University and conducted in accordance with the Declaration of Helsinki. The informed written consents were collected from every patient prior to tissue collection.

\section{Cell Transfection and Treatments}

HCC cell lines (Huh7, SMMC-7721, HepG2, Hep3B, and BEL-7404) and immortalized normal liver cell line LO2 were purchased from the American Type Culture
Collection (ATCC, Manassas, VA, USA). All cells were maintained with RPMI-1640 medium (Gibco, Grand Island, NY, USA) supplemented with 10\% FBS (Thermo Fisher Scientific, Inc., Waltham, MA, USA), and fostered in a humidified incubator containing $5 \% \mathrm{CO}_{2}$. When growing to $70-80 \%$ confluence, SMMC7721 and HepG2 cells were transfected with miR-137 mimics (miR-137), miR-137 antagomir (anti-miR-137), respective negative controls (miR-NC, anti-miR-NC), or miR-137 + Lv-DNMT3a (GenePharma Shanghai, China) with Lipofectamine 3000 reagent (Invitrogen, Carlsbad, CA, USA). The transfected cells were harvested for further analyses.

\section{Quantificational Real-Time Polymerase Chain Reaction (qRT-PCR)}

TRIzol reagent (Invitrogen) was used to isolate total sample RNAs. The miScript reverse transcription kit (Bio-Rad Laboratories, Hercules, CA, USA) was used to synthesize the single strand cDNA. TaqMan MicroRNA Assay kit (Applied Biosystems, Foster City, CA, USA) was used to examine miR-137 expression. To determine DNMT3a mRNA expression, Scientific RevertAid First Strand cDNA Synthesis Kit (Thermo Fisher Scientific) was applied to synthesize cDNA. SYBR Premix Ex Taq II (Takara, Dalian, China) was used to carry out the subsequent qPCR analyses. StepOnePlus real-time PCR system (Applied Biosystems) was used to monitor all the reactions. The U6 snRNA and GAPDH were employed as internal references for miR-137 and DNMT3a, respectively. The $2^{-\Delta \Delta \mathrm{Ct}}$ method was used to compute the relative gene expression. Each experiment was repeated independently at least 3 times.

\section{Western Blot}

Complete protease inhibitor Cocktail (Roche, Guangzhou, China) supplemented RIPA lysis buffer (Beyotime, Shanghai, China) was used to isolate sample proteins. Bicinchoninic acid protein assay kit (Peirce, Rockford, IL, USA) was employed to detect the concentration of total proteins. The extracted proteins were subjected to $10 \%$ SDS-PAGE, followed by electroblotting onto PVDF membranes (Millipore, Billerica, MA, USA). Blocked with $5 \%$ skimmed milk at $25^{\circ} \mathrm{C}$ for $2 \mathrm{~h}$, the blots were incubated with the respective primary antibodies at $4{ }^{\circ} \mathrm{C}$ overnight. Then, the blots were probed with corresponding HRP-conjugated secondary antibodies. Last, Enhanced Chemiluminescence Western 
Blotting kit (Peirce) was used to visualize the immunoreactive protein bands. The following primary antibodies were used in this section: DNMT3a (Bioss antibodies, Woburn, MA, USA), PCNA, caspase-3, cleaved caspase3, MMP-2, and MMP-7 (Abcam, Cambridge, UK); PTEN, Akt, p-Akt, and $\beta$-actin (Cell Signaling Technology, Beverly, MA, USA). Each experiment was repeated independently at least 3 times.

\section{Luciferase Reporter Assay}

PCR was used to synthesize 3'UTR of DMT3a where rested the putative miR-137 binding sites (DMT3a-WT) and its mutant (DMT3a-MUT). The pGL3-reporter luciferase vectors (Promega, Madison, WI, USA) were used to load the synthesized sequences. Next, miR-137, anti-miR-137, or respective controls and luciferase reporter constructs were co-transfected into SMMC7721 and Huh7 cells using Lipofectamine 3000 reagent (Invitrogen). Forty-eight hours post-delivery, Dual-Luciferase Reporter Assay System (Promega) was used to detect the luciferase activities. Each experiment was repeated independently at least 3 times.

\section{MTT Assay}

MTT assay was used to evaluate cell proliferative abilities. After transfection for $0,1,2$ and 3 days, cells were added with $20 \mu \mathrm{L}$ of MTT reagent $(5 \mathrm{mg} / \mathrm{mL}$; Sigma, St Louis, MO, USA). After $4 \mathrm{~h}$ incubation, $150 \mu \mathrm{L}$ of DMSO was used to dissolve formazan in the cells. Last, a Microplate Reader (Bio-Tek, Winooski, VT, USA) was used to read the optical density at $490 \mathrm{~nm}$ wavelength. Each experiment was repeated independently at least 3 times.

\section{Cell Apoptosis Assay}

At $48 \mathrm{~h}$ post-treatment, the harvested cells were resuspended with binding buffer. And the cells were stained with Annexin V-fluorescein isothiocyanate and propidium iodide (BD Biosciences, San Diego, CA, USA) in the dark for $15 \mathrm{~min}$. Then, a flow cytometer (FACSCalibur, BD Biosciences) was applied to assess the apoptotic cells. Each experiment was repeated independently at least 3 times.

\section{Wound Healing Assay}

Wound healing assay was used to assess cell migratory ability. The treated cells were cultured to reach $80 \%$ confluence. A $200 \mu \mathrm{L}$ sterile pipette tip was used to produce a uniform scratch on the cell monolayers. The generated scratch was photographed at 0 and $24 \mathrm{~h}$ with an inverse microscope (Olympus, Tokyo, Japan). Each experiment was repeated independently at least 3 times.

\section{Transwell Assay}

The Transwell chambers $(8-\mu \mathrm{m}$ pore size, Corning Incorporated, Corning, NY, USA) pre-coated with Matrigel (BD Biosciences) were used in this section. Upper compartments were added with cells suspended in $200 \mu \mathrm{L}$ serumfree medium, and the lower ones were added with $600 \mu \mathrm{L}$ medium supplemented with 10\% FBS. Forty-eight hours post-incubation, the invaded cells in the lower compartment were fixed with ethanol and stained using crystal violet for $30 \mathrm{~min}$. Last, an inverse microscope (Olympus) was used to count and record the invaded cells. Each experiment was repeated independently at least 3 times.

\section{Methylation-Specific PCR (MSP)}

Get pure DNA Kit (Dojindo Molecular Technologies, Gaithersburg, MD, USA) was used for extracting the cell genomic DNA. The bisulfite modification of genomic DNA for methylation analysis was conducted using an EpiTect Bisulfite kit (Qiagen Inc., Valencia, CA, USA). Then, the PTEN promoter methylation status was assessed using the EZ DNA Methylation Direct kit (Zymo Research, Orange, CA, USA) under the LightCycler 480 system (Roche Applied Science, Penzberg, Germany). Agarose gel electrophoresis was used to separate the PCR products and nucleic acid silver staining kit (Beijing Dingguo Changsheng Biotechnology Co., Ltd., Beijing, China) was used to stain them. Sequences for the methylated and unmethylated primers were listed as below: methylated PTEN, forward 5'TTCGTTCGTCGTCGTCGTATTT-3'， 5'-GCCGCTTAAC TCTAAACCGCAA-3'; unmethylated PTEN, forward 5'GTGTTGGTGGAGGTAGTTGTTT-3', reverse 5'-ACCA CTTAACTCTAAACCACAACCA-3'. Each experiment was repeated independently at least 3 times.

\section{Xenograft Nude Mouse Models}

Animal experiments strictly followed the Principles of Animal Care and Use of the First Affiliated Hospital of Zhengzhou University and were approved by the Ethics Committee of First Affiliated Hospital of Zhengzhou University. 9 nude mice (BALB/C, 6 weeks old, male, Slac Laboratory Animal Co. Ltd, China) were used to construct xenograft mouse models with right flank region subcutaneously injected with the transfected cells. The xenograft mouse models were sacrificed 4 weeks after inoculation. Xenografts were resected, pictured and 
measured. Tumor volume was calculated according to the formula: Volume $=\left(\right.$ length $\times$ width $\left.^{2}\right) / 2$. Each experiment was repeated independently at least 3 times.

\section{Immunohistochemistry (IHC) Assay}

Specimens were immersed in hydrogen peroxide at $37^{\circ} \mathrm{C}$ for $10 \mathrm{~min}$, and blocked with goat serum at $25^{\circ} \mathrm{C}$ for 2 h. Then, specimens were probed with the primary (DNMT3a) and secondary antibodies strictly followed the manufacture's instruction. Last, 3,3'diaminobenzidine (DAB) and hematoxylin were used to visualize the specimens. A light microscope (Olympus) was used for imaging the stained sections. DNMT3a expression was scored and the formula to calculate score was as follows: 4 (High Positive), 3+ (Positive), 2+ (Low Positive) and 1+ (Negative) based on the percentage of positive cells stained. Each experiment was repeated independently at least 3 times.

\section{Statistical Analysis}

SPSS17.0 software (IBM, Chicago, IL, USA) was employed for the statistics. The measurement data were shown as mean \pm standard deviation (SD) and analyzed by Student's $t$-test or analysis of variance (ANOVA). The counting data were analyzed with the Chi-square test. $P<0.05$ was rendered statistically significant.

\section{Results}

\section{A Decrease of miR-137 and an Increase of DNMT3a Were Observed in HCC Tissues and Cells}

The miR-137 and DNMT3a expression profiles in HCC tissues and cells were determined with Western blot, immunohistochemistry and qRT-PCR analyses. The miR-137 expression was significantly lowly expressed (Figure 1A), while DNMT3a mRNA expression (Figure 1B) and DNMT3a protein expression were dramatically upregulated (Figure 1C) in HCC tissues with respective to that in their healthy counterparts. DNMT3a expression status (IHC score $2+$ ) was found in $75 \%$ of HCC tissues and DNMT3a expression status (IHC score 1) was detected in $80 \%$ of healthy counterparts (data not shown). Further, a negative correlation between the expression of miR-137 and DNMT3a in HCC tissues was described by Spearman correlation analysis (Figure 1D). Meanwhile, miR-137 expression was also abnormally suppressed (Figure 1E), while both DNMT3a mRNA and protein levels were exceptionally higher (Figure $1 \mathrm{~F}$ and G) in HCC cell lines (Hep3B, SMMC-7721, HepG2, Huh7, and BEL-7404) relative with that in immortalized normal liver cell line LO2. Meanwhile, the analyses of miR-137 expression and the clinicopathological characteristics of HCC patients (Table 1) implicated a great significance of the correlation between the decreased miR-137 and tumor size
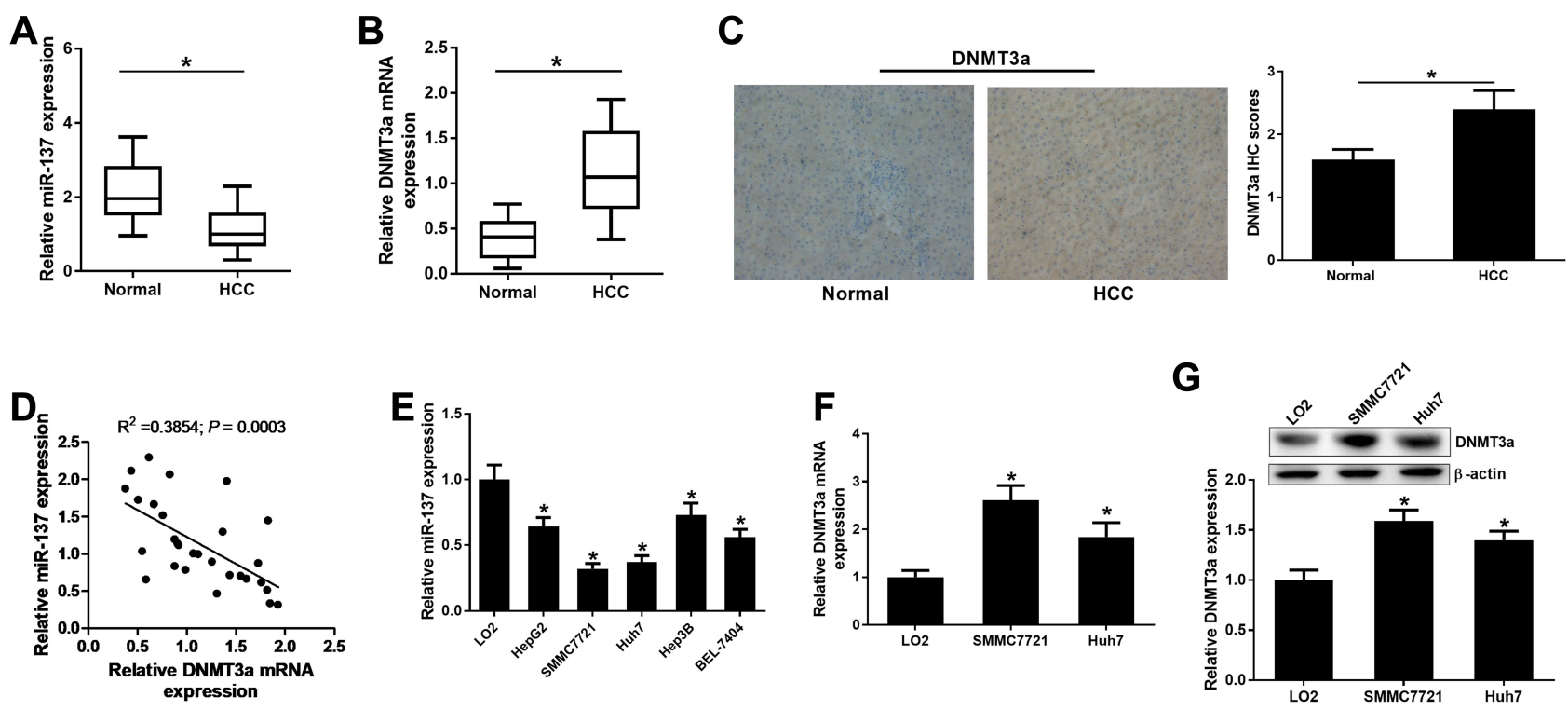

Figure I miR-I37 and DNMT3a expression in HCC clinical specimens and cells. qRT-PCR analyses for miR-I37 (A) and DNMT3a were (B) expressions in 29 paired HCC and their healthy counterparts. (C) Representative pictures (left) of IHC staining for DNMT3a expression and DNMT3a IHC scores (right) in HCC tissues and their healthy counterparts. (D) miR-137 was inversely correlated with DNMT3a mRNA level in HCC tissues. (E) qRT-PCR analyses for miR-I 37 level in different cell lines (Hep3B, HepG2, SMMC-772I, Huh7, and BEL7404, and LO2). (F and G) DNMT3a mRNA and protein levels in HCC cell lines (SMMC-772I, Huh7, and LO2) were valued using qRT-PCR and Western blot, respectively. *P < 0.05. 
Table I Relationship Between miR-137 Expression and Clinicopathological Features of HCC Patients

\begin{tabular}{|c|c|c|c|c|c|c|}
\hline \multicolumn{2}{|l|}{ Features } & \multirow[t]{2}{*}{$\mathbf{n}$} & \multicolumn{2}{|c|}{$\begin{array}{l}\text { miR-137 } \\
\text { Expression }\end{array}$} & \multirow[t]{2}{*}{$P$ value } & \multirow[t]{2}{*}{$\chi^{2}$} \\
\hline & & & High & Low & & \\
\hline Age & $\begin{array}{l}<50 \\
\geqq 50\end{array}$ & $\begin{array}{l}16 \\
13\end{array}$ & $\begin{array}{l}7 \\
5\end{array}$ & $\begin{array}{l}9 \\
8\end{array}$ & 0.774 & 0.083 \\
\hline Gender & $\begin{array}{l}\text { Male } \\
\text { Female }\end{array}$ & $\begin{array}{l}12 \\
17\end{array}$ & $\begin{array}{l}6 \\
6\end{array}$ & $\begin{array}{l}6 \\
11\end{array}$ & 0.428 & 0.627 \\
\hline $\begin{array}{l}\text { Tumor size } \\
(\mathrm{cm})\end{array}$ & $\begin{array}{l}<5 \\
\geqq 5\end{array}$ & $\begin{array}{l}13 \\
16\end{array}$ & $\begin{array}{l}8 \\
4\end{array}$ & $\begin{array}{l}5 \\
12\end{array}$ & 0.047 & 3.948 \\
\hline Liver cirrhosis & $\begin{array}{l}\text { Yes } \\
\text { No }\end{array}$ & $\begin{array}{l}22 \\
7\end{array}$ & $\begin{array}{l}8 \\
4\end{array}$ & $\begin{array}{l}14 \\
3\end{array}$ & 0.331 & 0.945 \\
\hline Metastasis & $\begin{array}{l}\text { Yes } \\
\text { No }\end{array}$ & $\begin{array}{l}16 \\
13\end{array}$ & $\begin{array}{l}4 \\
8\end{array}$ & $\begin{array}{l}12 \\
5\end{array}$ & 0.047 & 3.948 \\
\hline TNM stage & $\begin{array}{l}\text { I/II } \\
\text { III/IV }\end{array}$ & $\begin{array}{l}13 \\
16\end{array}$ & $\begin{array}{l}9 \\
3\end{array}$ & $\begin{array}{l}4 \\
13\end{array}$ & 0.006 & 7.535 \\
\hline $\mathrm{HBsAg}$ status & $\begin{array}{l}\text { Positive } \\
\text { Negative }\end{array}$ & $\begin{array}{l}19 \\
10\end{array}$ & $\begin{array}{l}8 \\
4\end{array}$ & $\begin{array}{l}11 \\
6\end{array}$ & 0.913 & 0.012 \\
\hline
\end{tabular}

(medium value, $P=0.047)$, metastasis $(P=0.047)$ and advanced TNM stage $(P=0.006)$. These speculated that there may be a close relationship between abnormal expressed miR-137 and DNMT3a in HCC progression and metastasis.

\section{miR- I37 Directly Targeted and Repressed DNMT3a in HCC Cells}

Aiming at exploring the miR-137 functions in HCC, miR137, anti-miR-137, or respective controls was transfected into SMMC7721 and Huh7 cells. As demonstrated by qRT-PCR assay, a notable increase of miR-137 expression was found after delivery of miR-137 (Figure 2A and B) in SMMC7721 and Huh7 cells. miR-137 was drastically decreased following introduction with anti-miR-137 in cells (Figure 2C and D). Interestingly, a dramatic reduction in DNMT3a after miR-137 delivery, while a noticeable elevation following anti-miR-137 delivery in HCC cells was revealed by Western blot analysis (Figure 2E and F). Furthermore, bioinformatics analyses by online software TargetScan showed that DNMT3A was a putative miR-137 target (Figure 2G). To verify the hypothesis, luciferase reporter assay was employed. As demonstrated by the luciferase reporter assay, introducing miR-137 greatly inhibited DNMT3a-WT luciferase activity in HCC cells, while inhibiting miR-137 conspicuously promoted (Figure 2H and I). However, both treatments failed to alter the luciferase activity in DNMT3a-MUT groups (Figure 2H and I). Thus, miR-137 interacting with DNMT3a directly in HCC cells was proved.

\section{miR-I37 Overexpression Resulted in} a Decrease in HCC Cell Proliferation and an Increase in Apoptosis by Decreasing DNMT3a Expression

To clarify whether miR-137 executed its biological function via DNMT3a, miR-137, miR-NC, or miR-137 + LvDNMT3a were delivered into SMMC7721 and Huh7 cells. The inhibitory effect of increased expression of miR-137 on DNMT3a expression was partly restored following reintroduction with Lv-DNMT3a in SMMC7721 and Huh7 cells (Figure 3A). MTT assays exhibited that upregulation of miR-137 distinctly impeded proliferation of SMMC7721 and Huh7 cells relative to control group, whereas this effect was effectively abolished by DNMT3a overexpression (Figure 3B). Also, flow cytometry analyses revealed a substantial enhancement in apoptosis in SMMC7721 (Figure 3C) and Huh7 (Figure 3D) following miR-137 overexpression, while augmented expression of DNMT3a partially attenuated this effect. Moreover, the protein expression of cell proliferation marker PCNA was apparently curbed in miR-137-overexpressing SMMC7721 (Figure 3E) and Huh7 (Figure 3F) cells. That effect of miR-137 overexpression was obviously reversed following DNMT3a overexpression. Furthermore, overexpression of miR-137 markedly increased cleaved caspase-3 level in SMMC7721 (Figure 3G) and Huh7 (Figure 3H) cells. However, those effects were remarkably reversed by forced expression of DNMT3a. However, miR-137 or DNMT3a overexpression produced little alternation in caspase-3 level. These data suggested that HCC cell proliferation was retarded and apoptosis was promoted because of the miR-137 overexpression induced DNMT3a inhibition.

\section{Enforced miR-I37 Expression Retarded HCC Cell Migratory and Invasive Traits by Decreasing DNMT3a Expression}

Next, the suppressive effect of miR-137 on HCC cell migratory abilities was revealed by wound healing assays (Figure 4A and B). The inhibitory roles of miR-137 in HCC cell invasion were also manifested by Transwell assays (Figure 4C and D). However, the above inhibitory effect was effectively reversed by ectopic DNMT3a expression (Figure 4A-D). MMPs are 
A

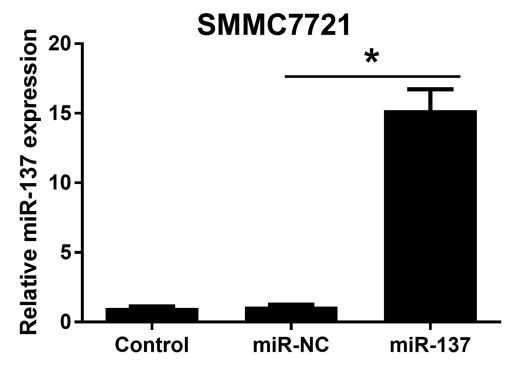

C

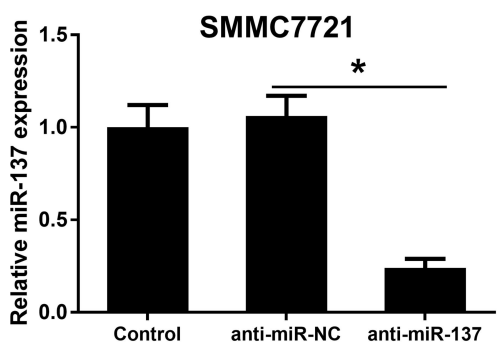

E
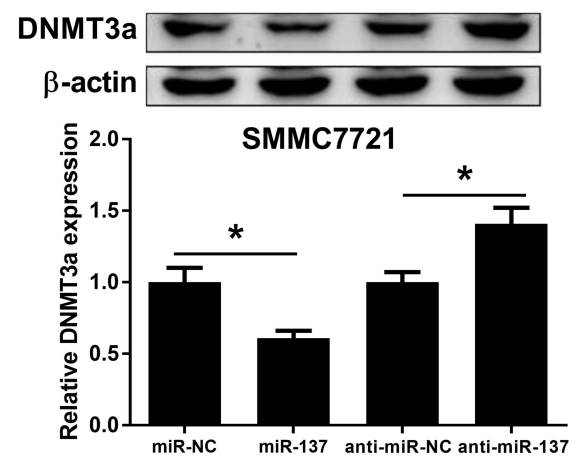

B

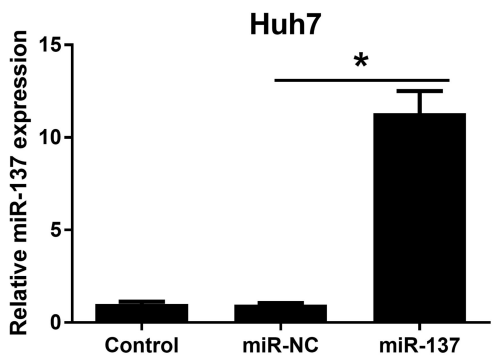

D

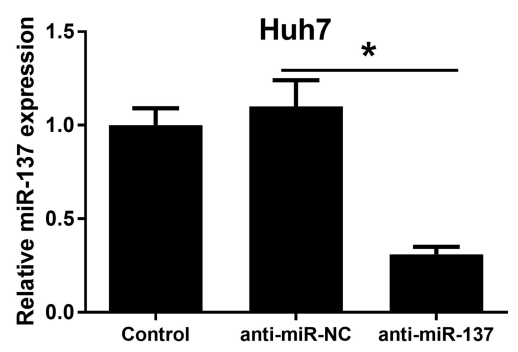

F DNMT3a $\beta$-actin

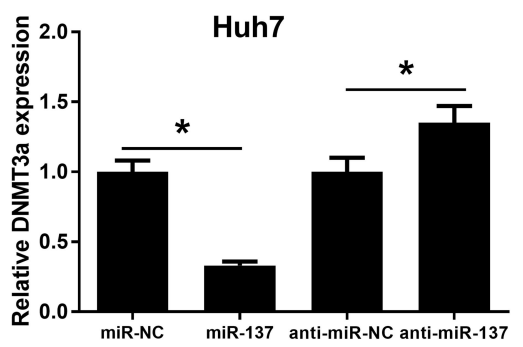

G Position 1111-1117 DNMT3a 3'UTR-WT 5'-...UUCAUCCAGACUCAUGCAAUAAC...-3' miR-137 3' GAUGCGCAUAAGAAUUCGUUAUU

DNMT3a-MUT $\quad 5^{\prime}-. . . U U C A U C C A G A C U C A U C U A C A A A C . . .-3^{\prime}$

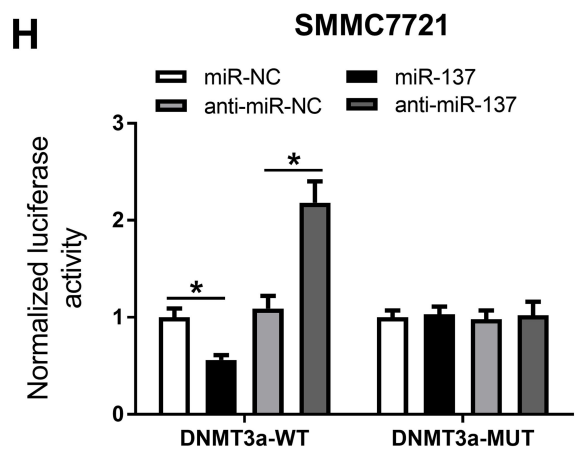

I

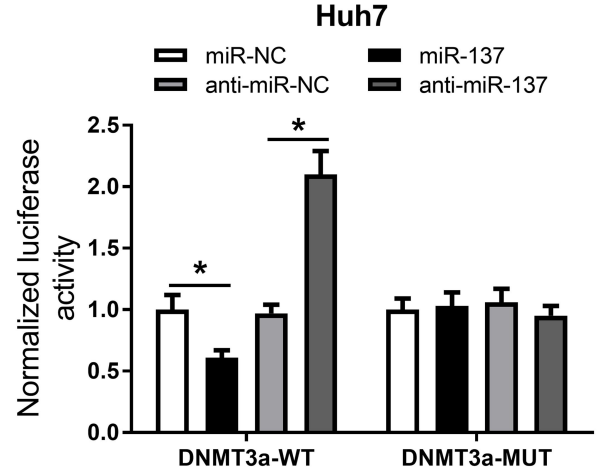

Figure 2 miR-137 targeted DNMT3a directly in HCC cells. miR-137 expression in SMMC772I (A) and Huh7 (B) cells received miR-NC or miR-I37 transfection, as well as SMMC772I (C) and Huh7 (D) cells received anti-miR-NC or anti-miR-137 infection. Western blot analyses for DNMT3a protein level in SMMC772I (E) and Huh7 (F) cells after delivery of miR-137, anti-miR-137, or corresponding controls. (G) The potential miR-137 targeting site in DNMT3a 3'UTR. Luciferase activity in SMMC772I (H) and Huh7 (I) cells after miR-137, anti-miR-137, or matched controls and DNMT3a-WT or DNMT3a-MUT co-transfection. *P $<0.05$. 
A
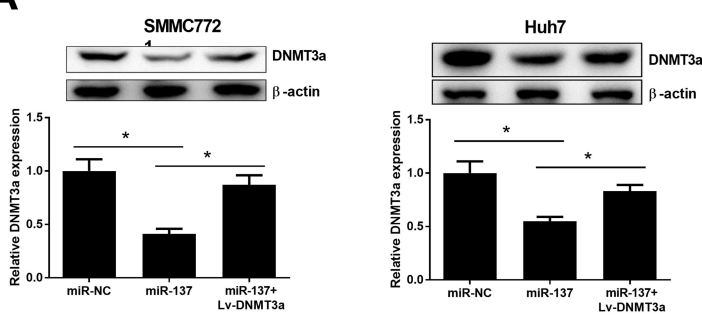

C
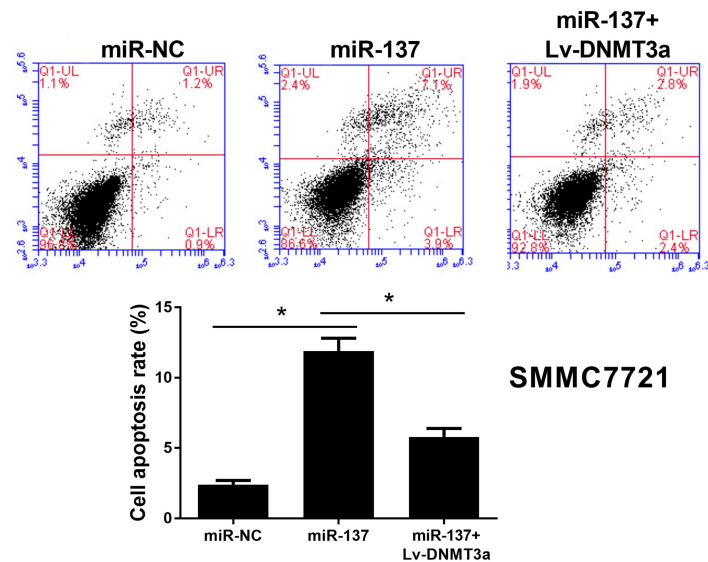

SMMC7721

E
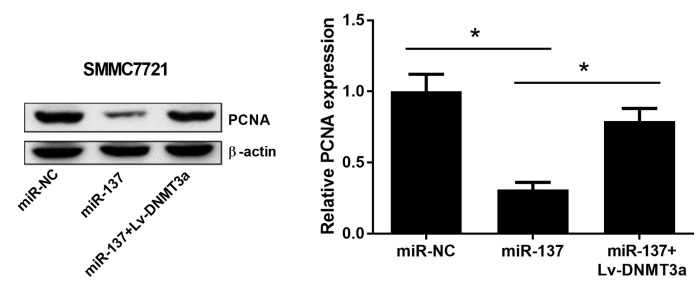

G
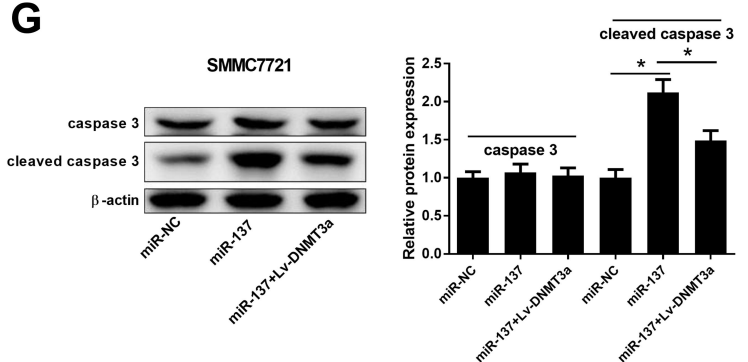

B
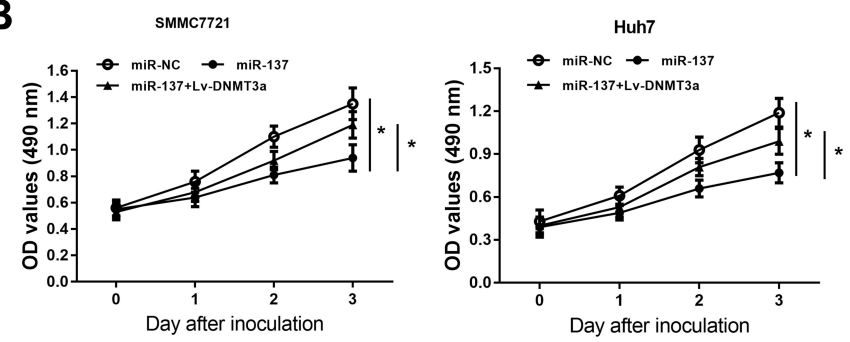

D
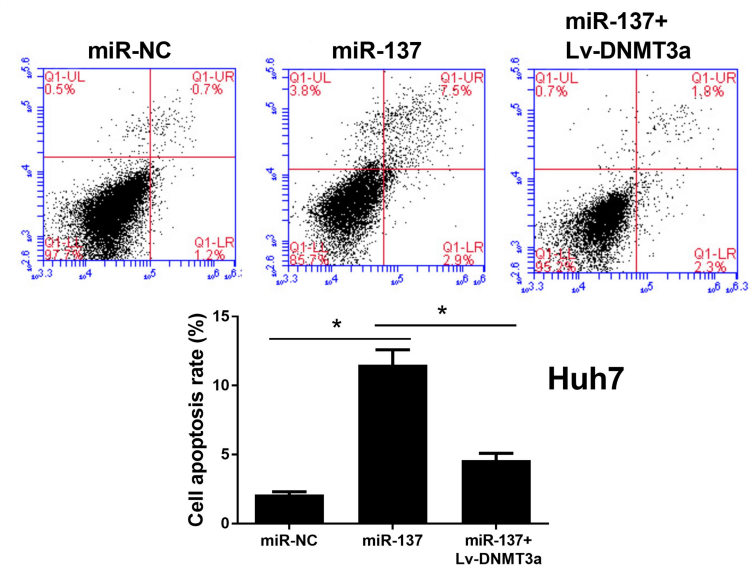

$\mathbf{F}$
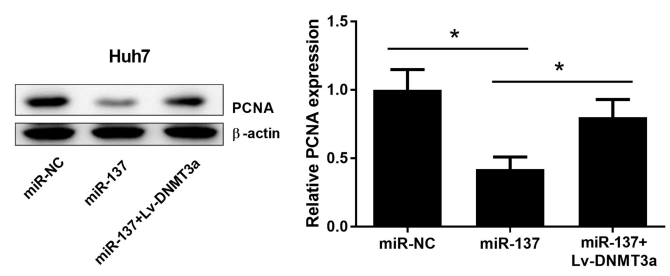

H
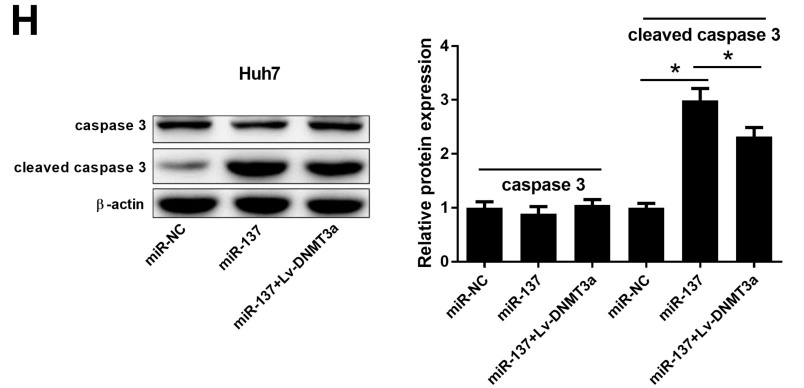

Figure 3 Effects of miR-I37 or combined with DNMT3a on cell proliferation and apoptosis in HCC cells. SMMC772I and Huh7 cells received miR-I37, miR-NC, or miR137 + Lv-DNMT3a delivery. (A) Western blot analyses for DNMT3a protein levels in the treated HCC cells. (B) MTT assays for cell proliferation of the treated HCC cells, at days 0, I, 2, and 3. (C and D) Flow cytometry analyses for apoptosis in the treated HCC cells. (E and F) Western blot analyses for PCNA protein levels in the treated HCC cells. ( $\mathbf{G}$ and $\mathbf{H}$ ) Western blot analyses for caspase- 3 and cleaved caspase-3 protein levels in the treated HCC cells. $* P<0.05$.

well known for participating in tumorigenesis regulation. ${ }^{13}$ The miR-137 overexpression resulted in a marked decrease of MMP-2 and MMP-7 levels, which was partially overturned following reintroduction with Lv-DNMT3a (Figure 4E and F). Together, the enforced miR-137 expression retarded HCC cell migratory and invasive abilities via interacting with DNMT3a.
miR-I37 Overexpression Inactivated the PTEN/Akt Pathway in HCC Cell by Decreasing DNMT3a Expression

The participation of PTEN/Akt signaling in HCC progression has been acknowledged in various studies. ${ }^{14,15}$ To confirm whether there was any biological regulation of miR-137 

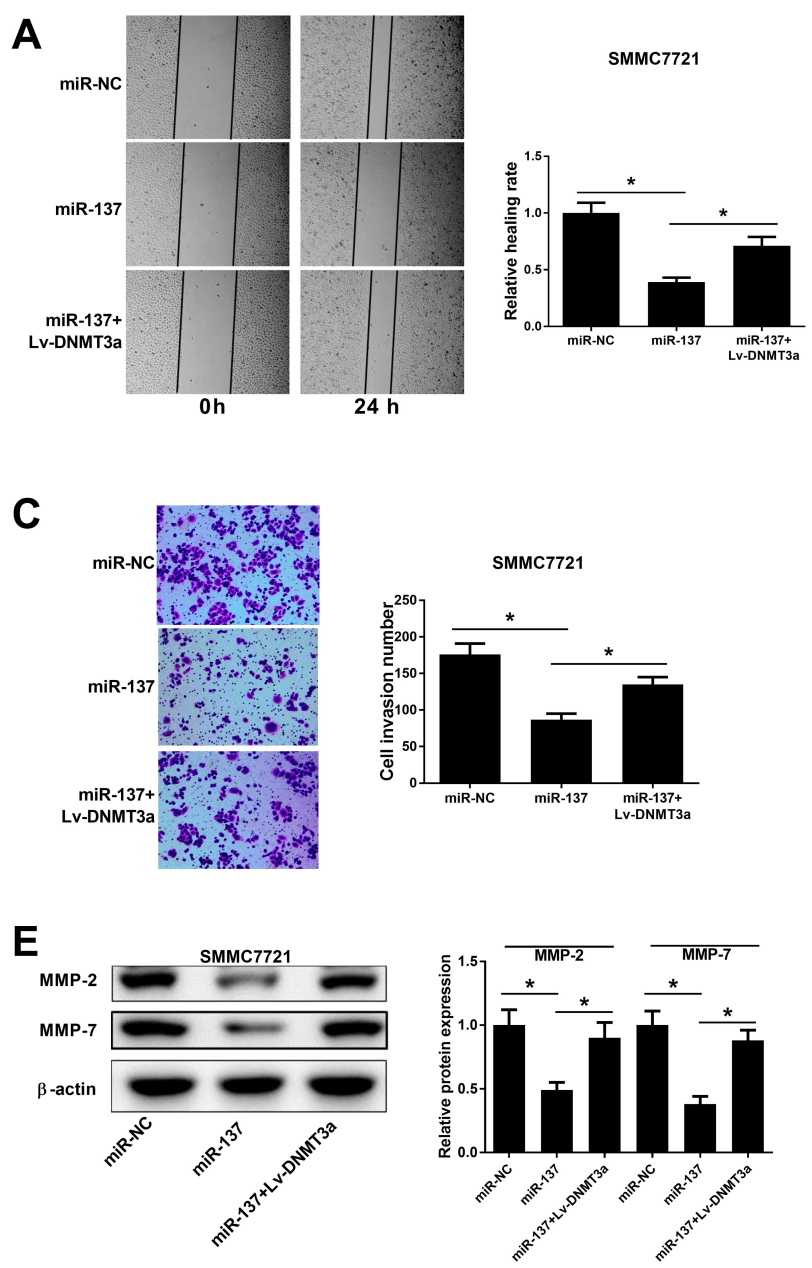
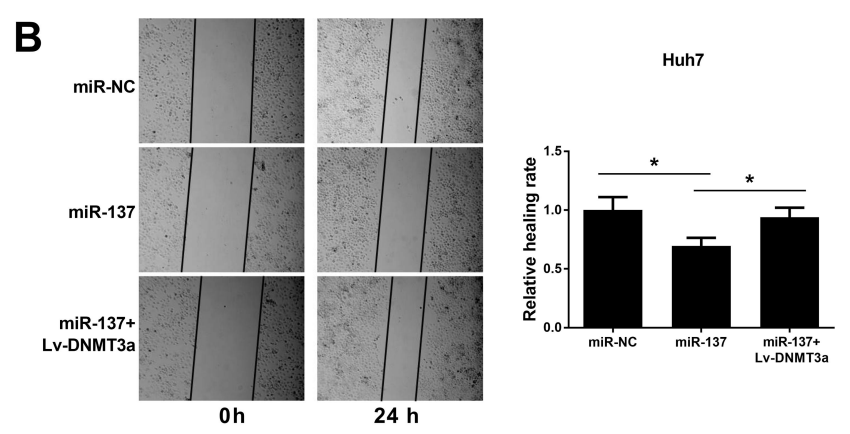

D
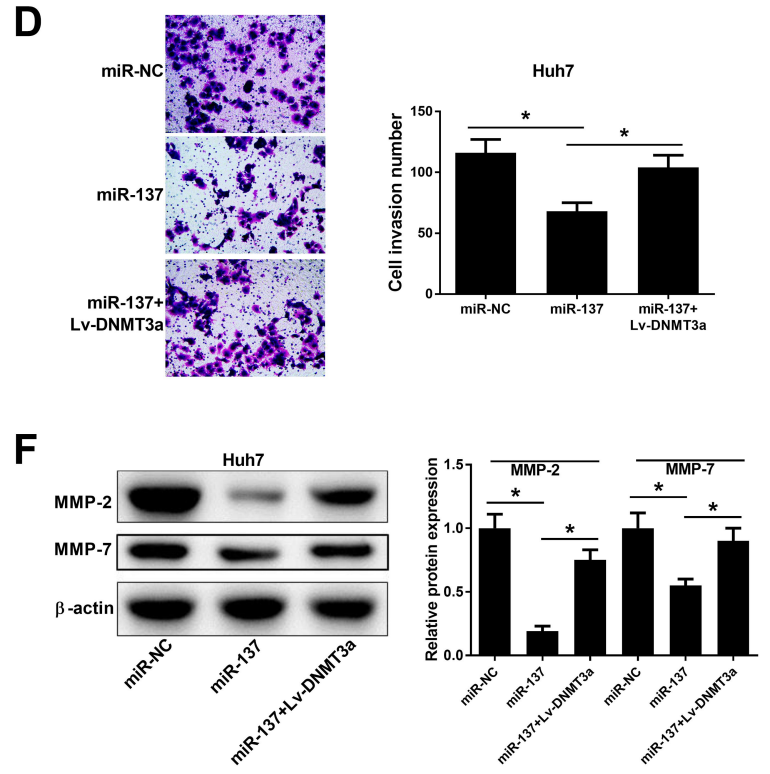

Figure 4 Effects of miR-I37 or combined with DNMT3a on cell migration and invasion in HCC cells. Cells were delivered with miR-I37, miR-NC, or miR-I37+Lv-DNMT3a. (A-D) Wound healing analyses and Transwell invasion analyses for HCC cell migratory and invasive abilities. (E and F) Western blot analyses for MMP-2 and MMP-7 protein levels in the transfected cells. $* P<0.05$.

on PTEN signaling in HCC cells, Western blot analysis was employed. The PTEN protein level was obviously increased upon miR-137 delivery, while DNMT3a overexpression considerably attenuated this effect in HCC cells (Figure 5A). Next, we further analyzed whether the increased PTEN expression was attributed to the demethylation of PTEN promoter region by miR-137. Moreover, the $\mathrm{CpG}$ sites of PTEN promoter were successfully demethylated following miR-137 overexpression in SMMC7721 and Huh7 cells, which was evidently recuperated by DNMT3a overexpression (Figure 5B and C). These results suggested that the increased PTEN expression was due to the demethylation of its $\mathrm{CpG}$ sites. Further, the involvement of the Akt pathway in miR-137 regulation on HCC cells was analyzed using Western blot. We observed an inhibition of Akt phosphorylation by miR-137 in HCC cells (Figure 5D and E), while this effect was partially restored by DNMT3a overexpression. These results manifested that miR-137 overexpression inactivated the PTEN/Akt pathway in HCC cells by decreasing DNMT3a expression.

\section{miR-I37 Overexpression Inhibited Tumor Growth in vivo in HCC via Interacting with DNMT3a and Inhibiting the PTEN/ Akt Cascades}

To verify the influence of miR-137 or along with DNMT3a in HCC in vivo, xenograft models were generated. Four weeks, mice were killed and xenografts were excised and analyzed. The tumors from the SMMC-7721miR-137 group presented significant decreased weight and volume compared with their counterparts, which was dramatically reversed following reintroduction with LvDNMT3a (Figure 6A-C). Moreover, IHC staining and 
A
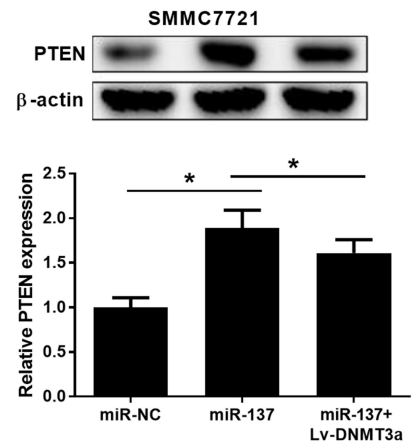

B

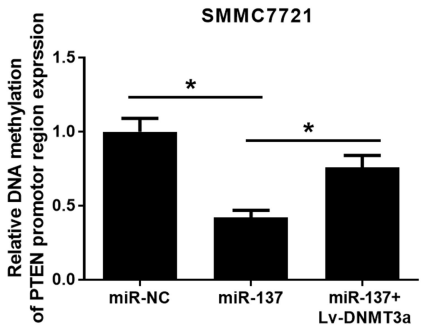

C

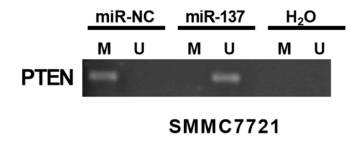

D

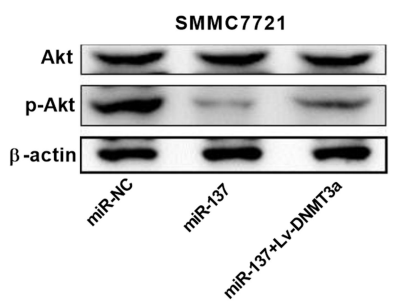

E

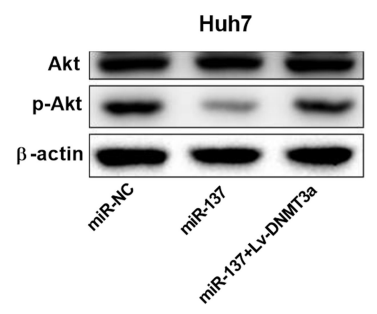

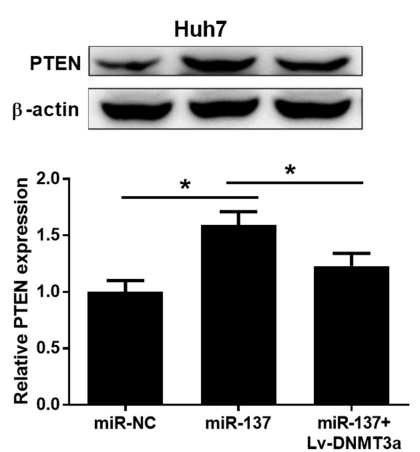
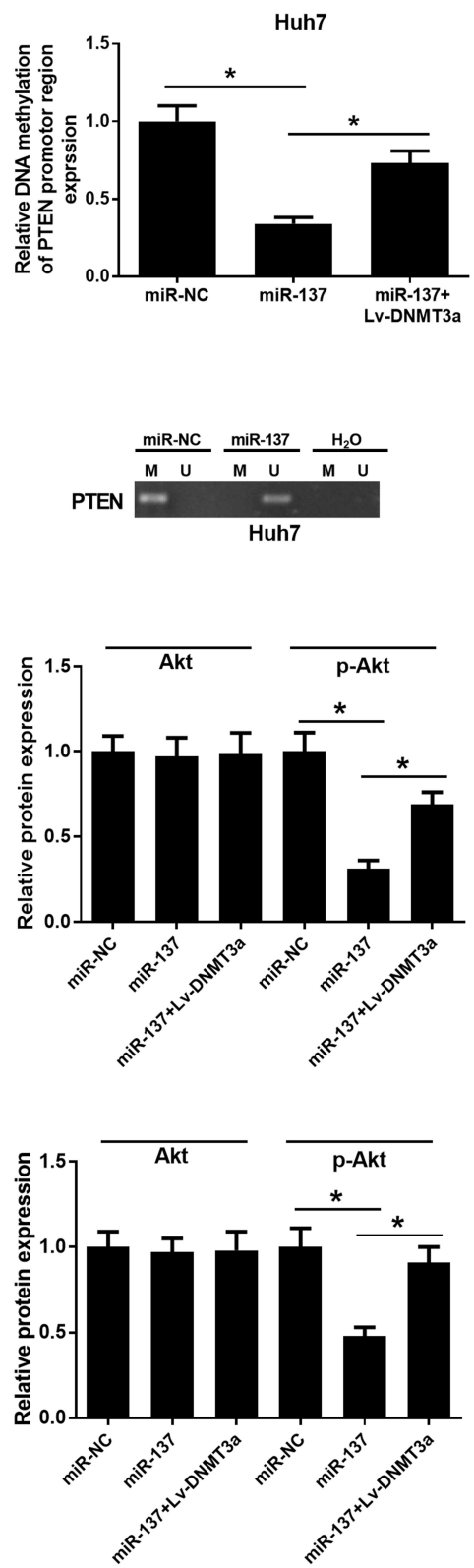

Figure 5 Effects of miR-I37 or combined with DNMT3a on the PTEN/Akt pathway in HCC cells. SMMC772I and Huh7 cells received miR-I37, miR-NC, or miR-I37 + LvDNMT3a transfection. (A) Western blot analyses for PTEN protein levels in the treated cells. (B and $\mathbf{C}$ ) MSP was conducted to examine the extent of methylation status of PTEN promoter region in the treated cells. ' $M$ ' and ' $U$ ' lanes represented PCR products obtained with methylation-specific and unmethylation-specific primers. $\mathrm{H}_{2} \mathrm{O}$ was used as a negative control. ( $\mathbf{D}$ and $\mathbf{E})$ Western blot analyses for Akt and $\mathrm{p}$-Akt protein levels in the treated $\mathrm{HCC}$ cells. $* \mathrm{P}<0.05$. 
A

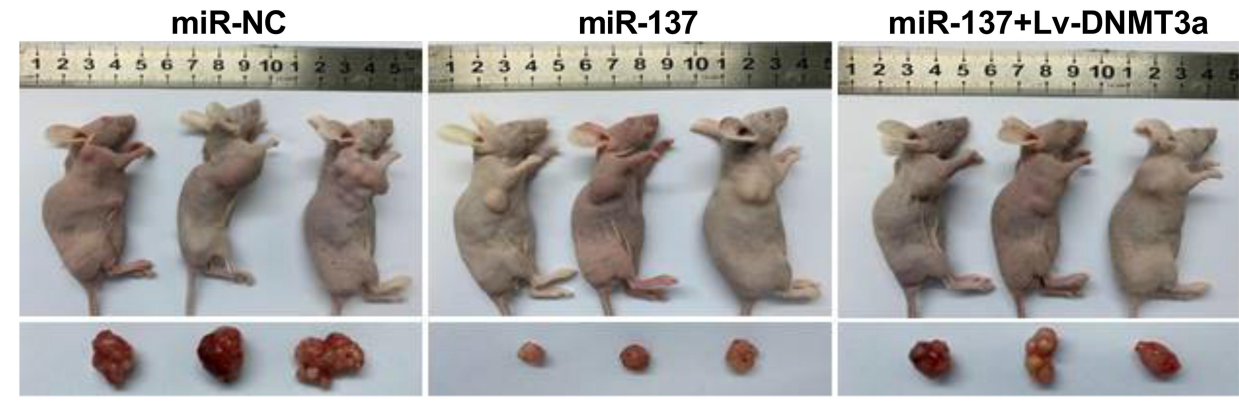

B

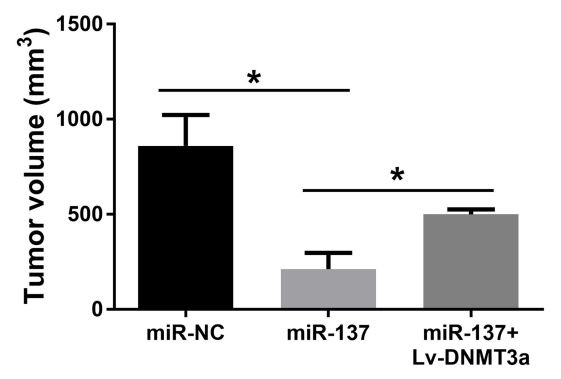

C

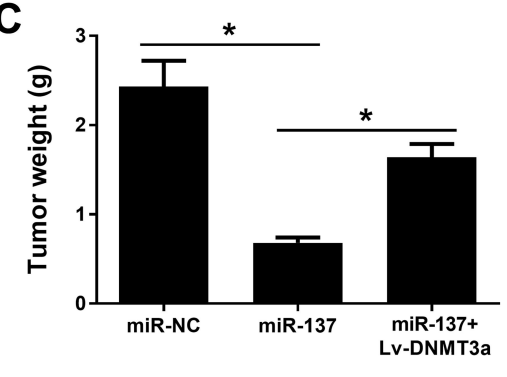

D

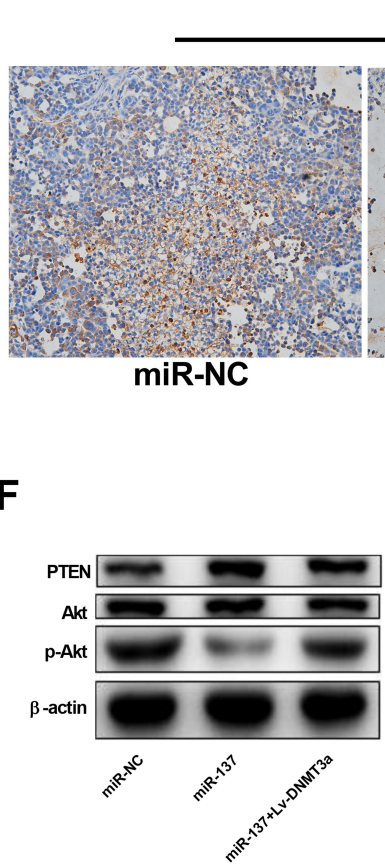

DNMT3a

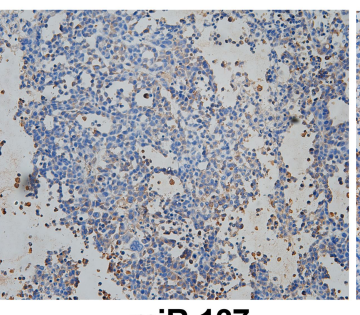

miR-137

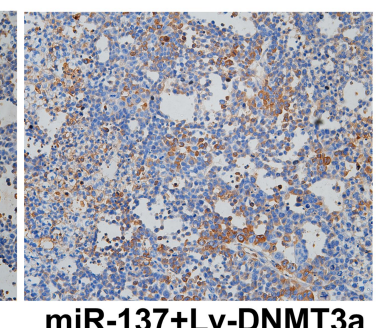

miR-137+Lv-DNMT3a
E
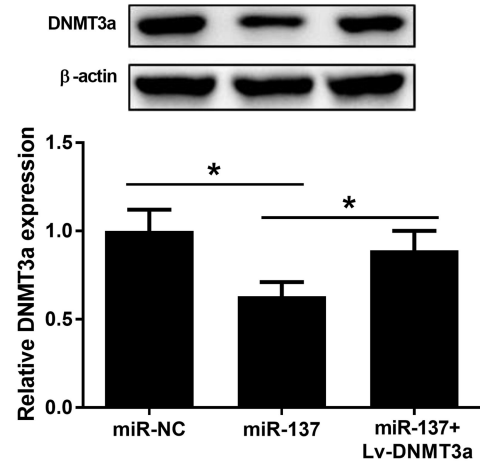

Figure 6 Effects of miR-I37 or combined with DNMT3a on HCC xenograft growth. The mice right flank regions were subcutaneously injected with miR-NC, miR-I37, or miR-I37 + Lv-DNMT3a introduced SMMC772I cells. (A) 4 weeks post-inoculation, tumor tissues were resected and pictured. Tumor volume (B) and weight (C) in different transfection groups. (D) DNMT3a expression in the xenografts by IHC staining. (E and F) Western blot analyses for DNMT3a, PTEN, p-Akt and Akt protein levels in the resected tumor tissues. $* P<0.05$.

Western blot assays showed that DNMT3a expression was markedly reduced in tumors overexpressing miR-137 (Figure 6D and E). And, those roles of miR-137 were abolished by overexpressing DNMT3a (Figure 6D and E). Furthermore, overexpressing miR-137 dramatically increased PTEN protein level and suppressed the phosphorylation of Akt in xenografted tumors (Figure 6F). However, the increased expression of DNMT3a drastically overturned those effects (Figure 6F). Collectively, these results suggested that miR-137 inhibited HCC tumor 
growth in vivo by targeting DNMT3a through suppressing the PTEN/Akt pathway.

\section{Discussion}

HCC belongs to the lethal human malignancies and leads to a huge burden on families and society due to its high metastasis and recurrence rate. ${ }_{-}^{1}$ It is widely believed that the abnormally expressed miRNAs are considered as important regulators in carcinogenesis. ${ }^{16}$ Hence, understanding miRNAs characteristics better would be helpful for understanding and controlling HCC better. Our study aimed at clarifying how miR-137 inhibited the HCC malignancy. Herein, we observed downregulation of miR-137 in HCC cells and tissues. DNMT3a was highly expressed in HCC cells and tissues and confirmed to be directly regulated by miR-137. miR-137 overexpression induced HCC cell apoptosis, as well as suppressed proliferative, migratory and invasive abilities via interacting with DNMT3a. Mechanistically, miR137 inactivated the PTEN/Akt cascades in HCC cells via inhibiting DNMT3a.

Recently, miR-137 has attracted increasing attention due to its tumor-suppressing effects by suppressing cell proliferation, migration, and promoting apoptosis in diverse malignancies. ${ }^{17-19}$ In contrast, miR-137 upregulation and functioning as an oncogene in bladder cancer and squamous cell carcinoma has been reported in recent studies. ${ }^{20,21}$ Here, we observed a decrease in miR-137 expression in HCC and demonstrated its suppressive effect on cell proliferative, migratory, and invasive abilities, as well as promotive effect on apoptosis, which was in accordance with the previous reports. ${ }^{12,22-24}$ More interestingly, our study found that miR-137 interacted with DNMT3a directly in HCC cells. The DNMT3a belongs to the DNA methyltransferases (DNMTs) family, which is crucial for carcinogenesis because of their epigenetic regulating abilities. ${ }^{25,26}$ Reportedly, DNMT3a is related to de novo methylation. ${ }^{27}$ As an oncogene, DNMT3a is ubiquitously overexpressed in many tumors such as pancreatic cancer, ${ }^{28}$ gastric cancer, ${ }^{29}$ and $\mathrm{HCC},{ }^{30}$ and implicated in tumorigenesis, differentiation and metastasis. ${ }^{31}$ Consistently, the overexpression of DNMT3a in HCC cells and tissues was observed in the present study. Moreover, the regulation of miR-137 overexpression on HCC cell proliferative, apoptotic, migratory and invasive capabilities was effectively abrogated by ectopic DNMT3a expression, suggesting that miR-137 overexpression inhibited HCC carcinogenesis via suppressing DNMT3a.
The PTEN, a well-studied tumor suppressor, regulates cell proliferation, survival, metastasis and energy metabolism. ${ }^{32}$ It is believed that the PTEN level is dependent on the methylation status of its promoter, which is priorly subjected to DNMT3a methylation. ${ }^{33}$ Promoter hypermethylation or silencing of PTEN is considered as the major mechanism for PTEN epigenetic inactivation. ${ }^{34}$ The aberrant DNA methylation induced PTEN silence has been well documented in many cancers including HCC. ${ }^{35}$ DNMT3a depletion inhibits HCC growth and progression via demethylating PTEN promoter. ${ }^{30}$ PTEN acts as a key inhibitor of the Akt pathway, ${ }^{36}$ and downregulation of PTEN induces hyperactivation of the Akt pathway, which contributes to carcinogenesis in various cancers. ${ }^{37}$ Our study proved that the $\mathrm{CpG}$ sites of PTEN promoter were successfully demethylated following restoration of miR-137 expression, thus resulting in increased PTEN expression. Furthermore, miR-137 overexpression ultimately inactivated the Akt pathway in HCC cells, which was mediated by downregulating DNMT3a. That miR-137 overexpression inhibited HCC carcinogenesis in vivo via interacting with DNMT3a and inhibiting the PTEN/Akt pathway has also been validated in our study.

\section{Conclusion}

In conclusion, our study demonstrated that the suppressive effect of miR-137 on HCC tumor growth was through targeting DNMT3a and suppressing the PTEN/Akt signaling. Also, targeting miR-137/DNMT3a/PTEN/Akt cascades may be a potential therapeutic strategy for HCC, which would be helpful for HCC control and treatment.

\section{Disclosure}

The authors declare no competing or financial interests.

\section{References}

1. Siegel RL, Miller KD, Jemal A. Cancer statistics, 2018. CA Cancer J Clin. 2018;68(1):7-30.

2. Zeng JY, Piao XH, Zou ZY, et al. Cryoablation with drug-loaded bead embolization in the treatment of unresectable hepatocellular carcinoma: safety and efficacy analysis. Oncotarget. 2018;9(7):75 57-7566. doi:10.18632/oncotarget.24029

3. X S Q, Guo XZ, Han GH, et al. MET inhibitors for treatment of advanced hepatocellular carcinoma: a review. World $J$ Gastroenterol. 2015;21(18):5445-5453. doi:10.3748/wjg.v21.i18.5445

4. J M Y, Sun LB, Zheng JS, et al. Copper chelation by trientine dihydrochloride inhibits liver RFA-induced inflammatory responses in vivo. Inflamm Res. 2016;65(12):1009-1020.

5. Hammond SM. An overview of microRNAs. Adv Drug Deliv Rev. 2015;87:3-14. doi:10.1016/j.addr.2015.05.001 
6. Gong J, He XX, Tian A. Emerging role of microRNA in hepatocellular carcinoma (Review). Oncol Lett. 2015;9(3):1027-1033. doi:10.3892/ol.2014.2816

7. Mizuguchi Y, Takizawa T, Yoshida H, et al. Dysregulated miRNA in progression of hepatocellular carcinoma: a systematic review. Hepatol Res. 2016;46(5):391-406. doi:10.1111/hepr.12606

8. Mao B, Wang G. MicroRNAs involved with hepatocellular carcinoma (Review). Oncol Rep. 2015;34(6):2811-2820. doi:10.3892/or.2015.4275

9. Smith AR, Marquez RT, Tsao W-C, et al. Tumor suppressive microRNA-137 negatively regulates Musashi-1 and colorectal cancer progression. Oncotarget. 2015;6(14):12558-12573. doi:10.18632/ oncotarget. 3726

10. Yang YR, Li YX, Gao XY, et al. MicroRNA-137 inhibits cell migration and invasion by targeting bone morphogenetic protein-7 (BMP7) in non-small cell lung cancer cells. Int J Clin Exp Pathol. 2015;8 (9):10847-10853.

11. Zhang W, Chen JH, Shan T, et al. miR-137 is a tumor suppressor in endometrial cancer and is repressed by DNA hypermethylation. $L a b$ Invest.2018;98(11):1397-1407.

12. Gao M, Liu L, Li S, et al. Inhibition of cell proliferation and metastasis of human hepatocellular carcinoma by miR-137 is regulated by CDC42. Oncol Rep. 2015;34(5):2523-2532. doi:10.3892/or.2015.4261

13. Zheng H, Takahashi H, Murai Y, et al. Expressions of MMP-2, MMP-9 and VEGF are closely linked to growth, invasion, metastasis and angiogenesis of gastric carcinoma. Anticancer Res. 2006;26 (5a):3579-3583.

14. Nathan N, Keppler-Noreuil KM, Biesecker LG, et al. Mosaic disorders of the PI3K/PTEN/AKT/TSC/mTORC1 signaling pathway. Dermatol Clin. 2017;35(1):51-60. doi:10.1016/j.det.2016.07.001

15. Lu M, Fei Z, Zhang G. Synergistic anticancer activity of 20 (S)-Ginsenoside $\operatorname{Rg} 3$ and Sorafenib in hepatocellular carcinoma by modulating PTEN/Akt signaling pathway. Biomed Pharmacother. 2018;97:1282-1288. doi:10.1016/j.biopha.2017.11.006

16. Calin GA, Croce CM. MicroRNA signatures in human cancers. Nat Rev Cancer. 2006;6(11):857-866. doi:10.1038/nrc1997

17. Zhang H, Yan T, Liu Z, et al. MicroRNA-137 is negatively associated with clinical outcome and regulates tumor development through EZH2 in cervical cancer. $J$ Cell Biochem.2018;119(1):938-947.

18. Du Y, Chen Y, Wang F, et al. miR-137 plays tumor suppressor roles in gastric cancer cell lines by targeting KLF12 and MYO1C. Tumour Biol. 2016;37(10):13557-13569. doi:10.1007/s13277-016-5199-3

19. Chen R, Zhang Y, Zhang C, et al. miR-137 inhibits the proliferation of human non-small cell lung cancer cells by targeting SRC3. Oncol Lett. 2017;13(5):3905-3911. doi:10.3892/ol.2017.5904

20. Xiu Y, Liu Z, Xia S, et al. MicroRNA-137 upregulation increases bladder cancer cell proliferation and invasion by targeting PAQR3. PLoS One. 2014;9(10):e109734. doi:10.1371/journal.pone.0109734

21. Wong TS, Liu XB, Wong BY, et al. Mature mir-184 as potential oncogenic microRNA of squamous cell carcinoma of tongue. Clin Cancer Res. 2008;14(9):2588-2592. doi:10.1158/1078-0432.CCR-07-0666

22. Zhu M, Li M, Wang T, et al. MicroRNA-137 represses FBI-1 to inhibit proliferation and in vitro invasion and migration of hepatocellular carcinoma cells. Tumour Biol. 2016;37(10):13995-14008. doi:10.1007/s13277-016-5230-8

OncoTargets and Therapy

\section{Publish your work in this journal}

OncoTargets and Therapy is an international, peer-reviewed, open access journal focusing on the pathological basis of all cancers, potential targets for therapy and treatment protocols employed to improve the management of cancer patients. The journal also focuses on the impact of management programs and new therapeutic

Submit your manuscript here: https://www.dovepress.com/oncotargets-and-therapy-journal
23. L L L, Lu SX, Li M, et al. FoxD3-regulated microRNA-137 suppresses tumour growth and metastasis in human hepatocellular carcinoma by targeting AKT2. Oncotarget. 2014;5(13):5113-5124. doi:10.18632/oncotarget.2089

24. Huang B, Huang M, Li Q. MiR-137 suppresses migration and invasion by targeting EZH2-STAT3 signaling in human hepatocellular carcinoma. Pathol Res Pract. 2018;214(12):1980-1986. doi:10.10 16/j.prp.2018.08.005

25. Huang Q, Ma C, Chen L, et al. Mechanistic insights into the interaction between transcription factors and epigenetic modifications and the contribution to the development of obesity. Front Endocrinol. 2018;9:370. doi:10.3389/fendo.2018.00370

26. B K O, Kim H, Park HJ, et al. DNA methyltransferase expression and DNA methylation in human hepatocellular carcinoma and their clinicopathological correlation. Int J Mol Med. 2007;20(1):65-73.

27. Hervouet E, Peixoto P, Delage-Mourroux R, et al. Specific or not specific recruitment of DNMTs for DNA methylation, an epigenetic dilemma. Clin Epigenetics. 2018;10:17. doi:10.1186/s13148-018$0450-\mathrm{y}$

28. He S, Wang F, Yang L, et al. Expression of DNMT1 and DNMT3a are regulated by GLI1 in human pancreatic cancer. PLoS One. 2011;6 (11):e27684. doi:10.1371/journal.pone.0027684

29. Yang J, Wei X, Wu Q, et al. Clinical significance of the expression of DNA methyltransferase proteins in gastric cancer. Mol Med Rep. 2011;4(6):1139-1143.

30. Zhao Z, Wu Q, Cheng J, et al. Depletion of DNMT3A suppressed cell proliferation and restored PTEN in hepatocellular carcinoma cell. J Biomed Biotechnol. 2010;2010:737535. doi:10.1155/2010/737535

31. Cao XY, Ma HX, Shang YH, et al. DNA methyltransferase3a expression is an independent poor prognostic indicator in gastric cancer. World J Gastroenterol. 2014;20(25):8201-8208. doi:10.3748/wjg. v20.i25.8201

32. Y R L, Chen M. The functions and regulation of the PTEN tumour suppressor: new modes and prospects. Nat Rev Mol Cell Biol. 2018;19(9):547-562. doi:10.1038/s41580-018-0015-0

33. Chik F, Szyf M. Effects of specific DNMT gene depletion on cancer cell transformation and breast cancer cell invasion; toward selective DNMT inhibitors. Carcinogenesis. 2011;32(2):224-232.

34. Luczak MW, Jagodzinski PP. The role of DNA methylation in cancer development. Folia Histochem Cytobiol. 2006;44(3):143-154.

35. Wang L, Wang WL, Zhang Y, et al. Epigenetic and genetic alterations of PTEN in hepatocellular carcinoma. Hepatol Res. 2007;37 (5):389-396. doi:10.1111/j.1872-034X.2007.00042.x

36. Maehama T, Dixon JE. PTEN: a tumour suppressor that functions as a phospholipid phosphatase. Trends Cell Biol. 1999;9(4):125-128. doi:10.1016/S0962-8924(99)01519-6

37. Buontempo F, Ersahin T, Missiroli S, et al. Inhibition of Akt signaling in hepatoma cells induces apoptotic cell death independent of Akt activation status. Invest New Drugs. 2011;29(6):1303-1313. doi:10. 1007/s10637-010-9486-3 agents and protocols on patient perspectives such as quality of life, adherence and satisfaction. The manuscript management system is completely online and includes a very quick and fair peer-review system, which is all easy to use. Visit http://www.dovepress.com/ testimonials.php to read real quotes from published authors. 